\title{
PROBABILIDAD DE PAGAR POR NOTICIAS DIGITALES EN ESPAÑA
}

\author{
Probability of paying for digital news in Spain
}

\section{Manuel Goyanes y Alfonso Vara-Miguel}

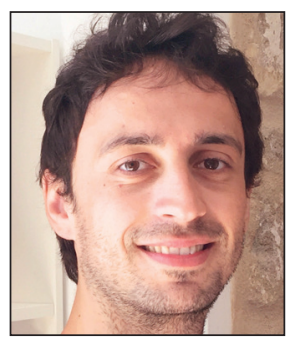

Manuel Goyanes es doctor en Ciencias de la Información por la Universidad de Santiago de Compostela y Master en Técnicas de investigación Cuantitativa por la Universidad Politécnica de Madrid. Es profesor e investigador en Gestión y sociología de la empresa informativa en la Universidad Carlos III de Madrid. Entre otros temas, ha escrito sobre liderazgo, disposición a pagar por información digital y sociología de las Ciencias de la Comunicación. Es autor del libro Desafío a la investigación estándar en comunicación. Críticas y alternativas (Editorial UOC).

http://orcid.org/0000-0001-6537-9777

Universidad Carlos III de Madrid, Facultad de Humanidades, Documentación y Comunicación, Departamento de Periodismo y Comunicación Audiovisual Calle Madrid, 133. 28903 Getafe (Madrid), España mgoyanes@hum.uc3m.es

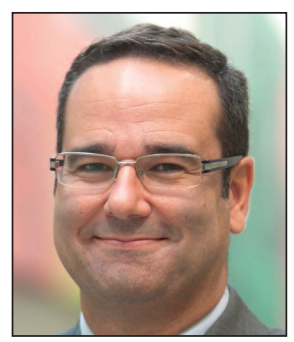

Alfonso Vara-Miguel es profesor de Economía, Periodismo económico y Empresa de comunicación en la Facultad de Comunicación de la Universidad de Navarra. Investigador del Center for Internet Studies and Digital Life (Cisdl). Su investigación reciente se ha centrado en los modelos de negocio de la industria periodística y en la cobertura informativa de la crisis económica.

http://orcid.org/0000-0003-4271-1858

Universidad de Navarra, Facultad de Comunicación, Departamento de Empresa Informativa. Campus Universitario, 31080 Pamplona, España avara@unav.es

\section{Resumen}

Se analiza la probabilidad de pagar por un diario digital en España. Mediante una encuesta a una muestra representativa de 2.104 españoles mayores de edad, mostramos, de modo general, el escaso número de ciudadanos dispuestos a pagar por información digital. Asimismo, a través de una regresión logística binomial exploramos los factores que afectan a esta probabilidad y discutimos las principales implicaciones prácticas para el desarrollo de estrategias de pago por contenidos de la industria de los periódicos.

\section{Palabras clave}

Pago por contenidos; Probabilidad de pago; Periódico digital; Interés en las noticias; Confianza en las noticias; Independencia de los medios de comunicación.

\section{Abstract}

The probability of paying for a digital newspaper in Spain is analyzed. Using a survey of a representative sample of 2,104 Spanish adults, we show, in general terms, the lack of people willing to pay for digital information. In addition, through a logistic regression analysis, we explore which factors affect this probability and discuss the main practical implications of developing paid-content strategies in the newspaper industry.

\section{Keywords}

Pay for content; Probability of paying; Digital newspapers; Cybermedia; Online newspapers; News interest; Trust in news; News independence.

Goyanes, Manuel; Vara-Miguel, Alfonso (2017). "Probabilidad de pagar por noticias digitales en España". El profesional de la información, v. 26, n. 3, pp. 488-496. 


\section{Introducción}

Como consecuencia de profundas transformaciones tecnológicas e industriales, las organizaciones periodísticas inician una etapa de exploración y ensayo de nuevos modelos de negocio (Goyanes, 2013), estrategias multi-plataforma (Schlesinger; Doyle, 2015) y estructuras organizativas (Campos-Freire, 2010) con las que adaptarse al nuevo entorno y ser rentables. A día de hoy, son muchas las causas que deterioran las cuentas anuales de los periódicos: caída de las ventas de publicidad y suscripciones impresas (SánchezTabernero, 2008), emergencia de nuevos competidores digitales (Picard, 2003), cambios en los patrones de consumo informativo (Huang, 2009; Vara-Miguel, 2015) y, sobre todo, la incapacidad generalizada de monetizar el contenido digital. Como respuesta a este último reto, muchos diarios han apostado por estrategias de pago por contenidos. En este contexto, la exploración de los factores que afectan la probabilidad de pagar por un periódico digital es un desafío estratégico para la supervivencia de la industria.

\section{Las organizaciones periodísticas están} en una etapa de exploración y ensayo de nuevos modelos de negocio

El objetivo de este estudio es explorar los factores que influyen en la probabilidad de pagar por un periódico digital en España. Diferentes investigaciones previas han analizado este aspecto (Cook; Attari, 2012; Chiou; Tucker, 2013; Chyi; Chadha, 2012; Goyanes, 2014; 2015; Berger et al., 2015; Arrese; Kaufmann, 2016), la mayoría de ellas mediante encuestas no representativas y además, restringidas a Estados Unidos. Por lo tanto, sólo podemos especular acerca de la generalización de los resultados a otros países, como España, donde el entorno tecnológico y competitivo que rodea a la industria de los periódicos es tan diferente ${ }^{1}$ (Goyanes; Dürrenberg, 2014). En este estudio tratamos de arrojar luz sobre este tema mediante una encuesta representativa a 2.104 españoles mayores de edad.

Nuestro estudio contribuye a la bibliografía en gestión de medios a través de una mejor comprensión del comportamiento de compra del consumidor digital, así como del desarrollo de una estratificación de los segmentos de mercado donde la probabilidad de pago es mayor. En este sentido, los resultados de la regresión logística binomial muestran cómo los más jóvenes ( $\mathrm{y}$ por lo tanto con mayor acceso y control de internet y las plataformas de pago) y con mayor educación e ingresos (capital cultural y económico) son los segmentos demográficos con mayor disposición a pagar por información digital. Del mismo modo, los perfiles de consumidor con mayor probabilidad de pago son aquellos que dentro de este segmento tengan un fuerte interés en las noticias, perciban un sistema de medios independiente de las influencias políticas y económicas (pero con escasa confianza en las noticias ("efecto tercera persona") y no utilicen Twitter para mantenerse informado ("efecto de sustitución"). La implementación de estrategias de pago por contenido en internet, enfatiza este artículo, se enfrenta a una poderosa cultura de lo gratis enraizada en la sociedad española: el $84 \%$ de los encuestados ( $N=1.858$ ) ha indicado que no ha pagado por acceder a un diario digital. El pago, en este contexto, continuaría siendo más un anhelo de las empresas de medios que una realidad factible para la sostenibilidad de los periódicos en el terreno digital.

El artículo se estructura del siguiente modo: inicialmente hacemos una revisión de la bibliografía previa sobre la probabilidad de pagar por información digital, y planteamos nuestras preguntas de investigación. A continuación, exponemos la metodología empleada. Una vez hecho esto, presentamos los resultados de la regresión logística binomial $y$, finalmente, discutimos los hallazgos fundamentales y las implicaciones teóricas y prácticas que se derivan de ellos.

Los más jóvenes (y por lo tanto con mayor acceso y control de internet y las plataformas de pago) y con mayor educación e ingresos (capital cultural y económico) tienen más disposición a pagar por información digital

\section{Estudios previos}

Diversas investigaciones de mercado han mostrado la escasa probabilidad de pagar por un periódico digital (KPMG, 2010; Newman; Levy, 2014; Newman et al., 2016). Algunos investigadores incluso han señalado que "es imposible cobrar por información general” (Herbert; Thurman, 2007, p. 15), que "la información debe ser gratuita a no ser que sea especializada" (Goyanes, 2013, p. 422) o que "esté firmada por un periodista de prestigio" (Filloux, 2011). Durante años, éstas han sido las tesis mantenidas a nivel académico y profesional, derivadas de los estudios de mercado y de las experiencias llevadas a cabo. Sin embargo, la dramática caída de ingresos por publicidad (PricewaterhouseCoopers, 2015) y la crisis estructural y coyuntural de los periódicos (Campos-Freire, 2010) han renovado el interés de la industria en el desarrollo de sistemas de ingresos basados en el pago del lector (Jenner; Fleming, 2011). En este contexto, son ya muchas las experiencias alrededor del mundo que muestran cómo monetizar relativamente el contenido online (Vara-Miguel et al., 2014; Arrese, 2016).

Sin embargo, por ahora, la fórmula exacta de esa rentabilidad está por descubrir. Porque convencer a un consumidor de pagar por información digital no resulta sencillo. Recientes encuestas de mercado así lo sugieren: únicamente el $3 \%$ de la circulación impresa es suscriptora digital, independientemente del precio del diario (American Press Institute; ITZBelden, 2009). Según la consultora KPMG (2010) el tipo de contenido online que los consumidores están más dispuestos a pagar es el mismo que normalmente pagan en soporte papel, pero únicamente el $2 \%$ pagaría si su diario favorito introdujera un muro de pago. En Estados Unidos sólo el $4 \%$ de los lectores paga por noticias digitales (Rosenstiel; Mitchell, 2011). Incluso en el exitoso caso de The New York Times, el millón de suscriptores digitales apenas representa el $2 \%$ de sus usuarios únicos mensuales (Holm, 2016). En España el pago a los medios se sitúa en torno al $10 \%$ de los 
internautas, después de un importante auge en 2015 (11\%) respecto a 2014 (8\%). Este porcentaje está ligeramente por debajo de la media del $13 \%$ de los 26 países incluidos en el estudio Digital news report 2016 (Newman et al., 2016) y muy alejado de Noruega (27\%), Brasil (22\%), Polonia y Suecia (20\%) (Vara-Miguel, 2016).

\section{Convencer a un consumidor a pagar por información digital no resulta sencillo: únicamente el $3 \%$ de la circulación im- presa es suscriptora digital}

Todos estos datos vienen a confirmar que a pesar de las facilidades de uso y consumo del diario online (acceso multiplataforma, contenido en constante actualización y consumo a cualquier momento del día), la mayoría de lectores evita el pago. Las razones de esta baja o nula disposición a pagar son múltiples y de diversa índole:

- consumo de una abundante oferta gratuita (Miguel de Bustos, 2010; Cea-Esteruelas, 2013; Himma-Kadakas; Kõuts, 2015)

- métodos de pago utilizados y su procedimiento de uso (Zhang; Nguyen, 2004);

- tipo de formato y plataforma de consumo (Chyi; Chadha, 2012);

- percepción de baja calidad del contenido ofertado (Himma-Kadakas; Kõuts, 2015; Peinado-Miguel; MateosAbarca, 2016); o

- creciente popularización de las redes sociales como fuentes gratuitas de información (Oh; Animesh; Pinsonneault, 2016).

De igual modo, las evidencias en relación con los factores que afectan a la probabilidad de pago por contenido online son múltiples. La mayoría de los estudios han analizado la influencia de variables sociodemográficas (sexo, edad, ingresos y educación), las relacionadas con el modo de informarse de los ciudadanos (frecuencia de consumo, soporte preferido, utilización de redes sociales para estar informado o la marca periodística), y el propio interés en las noticias.

Por lo que se refiere a las variables sociodemográficas, la mayoría de estudios han concluido que los hombres suelen tener mayor disposición a pagar por información digital de carácter generalista (Chyi; Chadha, 2012; Newman; Levy, 2014; Newman et al., 2015). En relación a la edad, diversos estudios han demostrado que los más jóvenes (por su pericia con las nuevas herramientas digitales y con las pasarelas de pago) suelen estar más inclinados a pagar por información (Pew Research Center, 2012; Chyi, 2012; Chyi; Lee, 2013; Goyanes, 2014; Punj, 2015). Sin embargo, son también los más jóvenes los más sensibles a la introducción de muros de pago, lo que tiende a provocar que desplacen su consumo hacia opciones gratuitas (Chiou; Tucker, 2013) o intenten acceder a las ediciones de pago aprovechando los resquicios (pequeñas artimañas) en la Web (Cook; Attari, 2012). Por lo que se refiere al nivel de ingresos, los resultados no son concluyentes. Algunos autores confirman una relación negativa entre ingresos e intención de pago por noticias digitales (Chyi; Chadha, 2012; Punj, 2015), otros una relación positiva (Goyanes, 2014) e incluso unos últimos han mostrado que el nivel de ingresos no influye en el pago por noticias digitales (Himma-Kadakas; Kõuts, 2015).

Al margen de los factores sociodemográficos, el segundo grupo de variables con las que predecir del pago por noticias digitales son aquellas relacionadas con el modo en que se informan los ciudadanos. Aquí se incluyen la suscripción a diarios impresos y la utilización de redes sociales. En primer lugar para la mayoría de autores la suscripción al diario impreso es un predictor significativo del pago por noticias digitales. De esta forma, las personas con una suscripción impresa suelen ser suscriptores o compradores de noticias digitales. Por otra parte, un uso moderado de Twitter es un predictor significativo de la intención de pago por noticias digitales (Goyanes, 2014). Finalmente, la mayor parte de los estudios ha demostrado que existe una relación entre el interés por las noticias y el pago por información digital (Chyi; Chadha, 2012; Chyi; Lee, 2013; Goyanes, 2014).

\section{A pesar de las facilidades de uso y consu- mo del diario online (acceso multi-plata- forma, contenido en constante actualiza- ción y consumo a cualquier momento del día), la mayoría de lectores evita el pago}

Dadas las múltiples alternativas de acceso a información gratuita y libre, los consumidores prefieren esas opciones antes que el pago a una suscripción a un periódico digital, lo que se traduce en dificultades de la industria en la monetización de los lectores digitales (Adams, 2012). Sin embargo, los factores que subyacen a esa escasa probabilidad de pago permanecen poco estudiados. Por lo tanto, con base en estudios previos, planteamos las siguientes preguntas de investigación:

PI1: ¿Cómo afectan las variables demográficas (sexo, edad, ingresos, educación y condición política) al pago por un periódico digital?

PI2: ¿Cómo afecta el interés en las noticias al pago por un periódico digital?

PI3: ¿Cómo afecta la confianza en las noticias al pago por un periódico digital?

PI4: ¿Cómo afecta la percepción de independencia de los medios de comunicación españoles al pago por un periódico digital?

PI5: ¿Cómo afecta la compra de un periódico impreso al pago por un periódico digital?

PI6: ¿Cómo afecta el uso de Facebook/Twitter como herramienta informativa al pago por un periódico digital?

\section{Metodología}

El análisis se basa en los datos correspondientes a España de la encuesta Digital news report 2016, en la que fueron incluidas varias preguntas directamente relacionadas con esta investigación ${ }^{2}$. El modelo construido en esta investigación se basa en una regresión logística binomial y analiza la probabilidad de pagar por información online como variable 
dependiente. La regresión logística mide la probabilidad de que un evento dicotómico ocurra, en este caso, la participación en una actividad de compra. La proporción de actividad predicha sigue el modelo logístico $\ln p /\left(1-p_{i}\right)=\beta X_{i}$, donde $p_{i}$ es la probabilidad de pagar por contenido online.

\subsection{Variable dependiente}

El pago por información digital ha sido medida como variable categórica dicotómica y codificada como 1 = Sí, he pagado por contenido online o accedido a un diario digital de pago el año pasado, y $0=$ No, no he pagado por contenido online o accedido a un diario digital de pago el año pasado.

Los hombres suelen tener mayor disposición a pagar por información digital de carácter generalista

\subsection{Variables independientes}

El sexo ha sido codificado como variable categórica dicotómica ( 1 = hombre; 2 = mujer), la edad como variable continua, al igual que los ingresos (brutos del hogar); la educación ha sido codificada como variable categórica politómica:

1 = actualmente estoy matriculado en el instituto;

2 = no completé los estudios secundarios o bachillerato;

3 = estudios secundarios o bachillerato;

4 = formación profesional;

5 = graduado;

6 = master $\mathrm{o}$ doctorado.

La condición política se ha codificado como 1 = izquierda; 2 = centro; 3 = derecha.

El interés en las noticias ha sido medido mediante la siguiente pregunta: "En general... cuánto dirías que estás interesado en las noticias" en una escala Likert 1-5 donde 1 $=$ no tengo ningún interés y $5=$ tengo un interés extremo.

La confianza en las noticias ha sido medida mediante el nivel de acuerdo o desacuerdo (en una escala Likert 1-5, donde 1 = totalmente en desacuerdo y 5 = totalmente de acuerdo) con el siguiente ítem: "Creo que puedo confiar en la mayoría de noticias la mayoría de las veces".

La independencia informativa frente a las influencias políticas y gubernamentales ha sido medida mediante el nivel de acuerdo o desacuerdo (en una escala Likert 1-5, donde 1 = totalmente en desacuerdo y $5=$ totalmente de acuerdo) con el ítem: "Los medios de comunicación en mi país son independientes de una indebida influencia política o gubernamental la mayoría de las veces".

La compra del periódico impreso ha sido medida como variable categórica dicotómica y codificada como 1 = Sí, he comprado un periódico impreso la semana pasada y 2 = No, no he comprado un periódico impreso la semana pasada.

Los consumidores prefieren las alternativas de acceso a información gratuita y libre antes que el pago de la suscripción a un periódico digital, lo que se traduce en dificultades de la industria en la monetización de los lectores digitales

Finalmente, el uso de Facebook o Twitter como medio informativo ha sido medido como variable categórica dicotómica mediante la pregunta: "Has usado Twitter/Facebook como herramienta para buscar, leer, ver, compartir o discutir contenido informativo la semana pasada?" y codificada como 1 = Sí, he usado Twitter/Facebook como... y 2 = No, no he usado Twitter/Facebook como...

\section{Resultados}

\subsection{Descriptivos}

La muestra representativa de 2.104 españoles está formada por un $53,7 \%$ ( $N=1.129)$ de hombres y un $46,3 \%$ de mujeres ( $N=975)$, de una edad entre los 18 y 94 años $(M=44,82$; DE: 14,67$)$. Las correlaciones y medias de las variables continuas y las categóricas ordinales se muestran en la tabla 1. Del total de personas encuestadas, únicamente el 10,2\% (N $=215$ ) ha pagado por acceder a un diario digital, mientras que el $88,4 \%(N=1.858)$ ha indicado que no (el $1,4 \%$ restante ha indicado que no sabe o no contesta).

Por otra parte, el 48,2\% ( $N=1.014)$ de los encuestados ha comprado un diario impreso la semana pasada frente al $50,6 \%$ ( $N=1.065$ ) que no. Por último, en cuanto al uso de Facebook y Twitter como fuentes de información, el $49 \%$ ( $N=$ 1.030) ha indicado que ha usado Facebook para buscar, leer, ver, compartir o discutir contenido informativo la semana pasada. Mientras, únicamente el 19,4\% ( $N=409)$ de los encuestados afirma que ha usado Twitter para informarse.

\subsection{Regresión logística}

Los resultados de la regresión logística muestran la relación

Tabla 1. Medias, desviaciones estándar y correlaciones de las variables continuas y categóricas ordinales

\begin{tabular}{|l|c|c|c|c|c|c|c|}
\hline \multicolumn{1}{|c|}{ Variable } & $\mathbf{M}$ & $\mathbf{D E}$ & Edad & Ingresos & Educación & $\begin{array}{c}\text { Interés noticias } \\
\text { Confianza } \\
\text { noticias }\end{array}$ & \begin{tabular}{c} 
Independencia \\
\hline Edad
\end{tabular} \\
\hline Ingresos & 44,82 & 14,67 & 1 & $0,129^{* *}$ & 0,042 & $0,067^{* *}$ & $0,122^{* *}$ \\
\hline Educación & 5,75 & 3,34 & $0,129^{* *}$ & 1 & $0,294^{* *}$ & $0,157^{* *}$ & 0,032 \\
\hline Interés noticias & 4,12 & 1,16 & 0,042 & $0,294^{* *}$ & 1 & $0,140^{* *}$ & $-0,006$ \\
\hline Confianza noticias & 4,16 & 0,69 & $0,067^{* *}$ & $0,157^{* *}$ & $0,140^{* *}$ & $-0,031$ \\
\hline Independencia & 2,20 & 1,08 & $0,122^{* *}$ & 0,032 & $-0,006$ & $0,112^{* *}$ & $0,078^{* *}$ \\
\hline
\end{tabular}


entre el pago por un periódico digital y variables predictoras ( $p<0,05$ ) como las demográficas (edad, ingresos y educación), el interés en las noticias, la confianza en las noticias, la independencia de los medios de comunicación españoles, el pago por un periódico impreso y el uso de Twitter como medio informativo (ver tabla 1). Sin embargo, variables independientes como el sexo, la condición política o el uso de Facebook como herramienta informativa no han resultado estadísticamente significativas. El último modelo explica el 10 ó el $21 \%$ de la varianza (Nagelkerke $R^{2}=0,212$; Cox and Snell $\left.R^{2}=0,106\right)$, sugiriendo un considerable poder de explicación.

Un uso moderado de Twitter es un predictor significativo de la intención de pago por noticias digitales

En relación con la primera pregunta de investigación, la influencia de variables demográficas en el pago por un diario digital, el sexo, la edad, los ingresos, la educación y la condición política fueron introducidos en el primer bloque de análisis. Los resultados muestran una asociación estadísticamente significativa $(p<0,05)$ con la edad (negativa), ingresos y educación, pero no con el sexo y la condición política. De esta forma, el pago por un diario digital $(\beta=-0,030$; $p$ $<0,05)$ es mayor en personas jóvenes. En otras palabras, el pago aumenta cuando la edad disminuye $\left(0,970 \mathrm{e}^{\mathrm{x}}\right)$. Por otra parte, las personas con mayores ingresos tienen más probabilidad de pago que los que tienen ingresos menores $\left(\beta=0,058 ; 1,060 e^{x} ; p<0,05\right)$, de igual modo que los que tienen un nivel educativo alto $\left(\beta=0,218 ; 1,243 e^{x} ; p<0,05\right)$ tienen más probabilidades de pago por un diario digital que los que tienen un nivel educativo menor.
La segunda pregunta de investigación cuestionaba cómo afecta el interés en las noticias al pago por un periódico digital. Además de la edad $\left(\beta=-0,031 ; 0,970 e^{x} ; p<0,05\right)$, los ingresos $\left(\beta=0,044 ; 1,045 e^{x} ; p<0,05\right)$, y la educación $\left(\beta=0,194 ; 1,214 e^{x} ; p<0,05\right)$, el interés en las noticias $(\beta=$ 0,$\left.720 ; 2,054 e^{x} ; p<0,05\right)$ también es un predictor positivo y significativo del pago por un periódico digital. De esta forma, el pago por un periódico digital es mayor si el interés en las noticias es alto.

\section{El estudio confirma que el pago por noti- cias digitales es minoritario entre los in- ternautas españoles $(10,2 \%)$ y que abun- da el consumo gratuito de información}

La tercera y cuarta pregunta de investigación cuestionaban cómo afecta la confianza en las noticias y la percepción de independencia de los medios de comunicación españoles al pago por un periódico digital. Los resultados de la regresión logística muestran que además de la edad $(\beta=-0,029 ; 0,972$ $\left.\mathrm{e}^{\mathrm{x}} ; \mathrm{p}<0,05\right)$, la educación ${ }^{3}\left(\beta=0,223 ; 1,250 \mathrm{e}^{\mathrm{x}} ; \mathrm{p}<0,05\right)$ y el interés en las noticias $\left(\beta=0,643 ; 1,903 e^{x} ; p<0,05\right)$, la confianza en las noticias $\left(\beta=-0,283 ; 0,754 e^{x} ; p<0,05\right)$ y la percepción de independencia de los medios de comunicación $\left(\beta=0,539 ; 1,714 \mathrm{e}^{\mathrm{x}} ; \mathrm{p}<0,05\right)$ son predictores significativos. La probabilidad de pago por un periódico digital aumenta si la confianza en las noticias disminuye, mientras que aumenta si la percepción de independencia de los medios de comunicación aumenta.

La quinta pregunta de investigación se cuestiona acerca de la relación entre la compra de un diario impreso y el pago a un diario digital. Además de la edad $\left(\beta=-0,031 ; 0,970 \mathrm{e}^{\mathrm{x}} ; \mathrm{p}\right.$ $<0,05)$, la educación $\left(\beta=0,215 ; 1,240 \mathrm{e}^{\mathrm{x}} ; \mathrm{p}<0,05\right)$, el inte-

Tabla 2. La última categoría (en las variables categóricas: sexo, pago impreso, Facebook y Twitter) es la tomada como referencia

\begin{tabular}{|c|c|c|c|c|c|c|c|c|c|c|}
\hline & \multicolumn{2}{|c|}{ Modelo 1} & \multicolumn{2}{|c|}{ Modelo 2} & \multicolumn{2}{|c|}{ Modelo 3} & \multicolumn{2}{|c|}{ Modelo 4} & \multicolumn{2}{|c|}{ Modelo 5} \\
\hline & $\beta$ & $\operatorname{Exp}(\beta)$ & $\beta$ & $\operatorname{Exp}(\beta)$ & $\beta$ & $\operatorname{Exp}(\beta)$ & $\beta$ & $\operatorname{Exp}(\beta)$ & $\beta$ & $\operatorname{Exp}(\beta)$ \\
\hline Sexo & 0,252 & 1,286 & 0,200 & 1,222 & 0,176 & 1,193 & 0,089 & 1,094 & 0,072 & 1,074 \\
\hline Edad & $-0,030^{* *}$ & 0,970 & $-0,031^{* *}$ & 0,970 & $-0,029^{* *}$ & 0,972 & $-0,031^{* *}$ & 0,970 & $-0,027^{* *}$ & 0,973 \\
\hline Ingresos & $0,058^{*}$ & 1,060 & $0,044^{*}$ & 1,045 & 0,039 & 1,039 & 0,024 & 1,024 & 0,023 & 1,023 \\
\hline Educación & $0,218^{* *}$ & 1,243 & $0,194^{* *}$ & 1,214 & $0,223^{* *}$ & 1,250 & $0,215^{* *}$ & 1,240 & $0,217^{*}$ & 1,242 \\
\hline Política & $-0,155$ & 0,857 & $-0,104$ & 0,901 & $-0,056$ & 0,946 & $-0,057$ & 0,944 & $-0,111$ & 0,895 \\
\hline Interés noticias & & & $0,720 * *$ & 2,054 & $0,643 * *$ & 1,903 & $0,481 * *$ & 1,618 & $0,410^{* *}$ & 1,508 \\
\hline Confianza noticias & & & & & $-0,283^{* *}$ & 0,754 & $-0,278^{* *}$ & 0,757 & $-0,296^{* *}$ & 0,744 \\
\hline Independencia & & & & & $0,539 * *$ & 1,714 & $0,450^{* *}$ & 1,568 & $0,469 * *$ & 1,598 \\
\hline Pago impreso & & & & & & & $-1,248^{* *}$ & 0,287 & $-1,279 * *$ & 0,278 \\
\hline Facebook & & & & & & & & & $-0,152$ & 0,859 \\
\hline Twitter & & & & & & & & & $-0,653^{* *}$ & 0,520 \\
\hline Constante & $-2,190^{* *}$ & 0,112 & $-5,107^{* *}$ & 0,006 & $-5,496^{* *}$ & 0,004 & $-3,845^{* *}$ & 0,021 & $-3,080^{* *}$ & 0,046 \\
\hline Nagelkerke $R^{2}$ & 0,063 & & 0,098 & & 0,152 & & 0,198 & & 0,212 & \\
\hline Cox \& Snell $R^{2}$ & 0,031 & & 0,049 & & 0,076 & & 0,099 & & 0,106 & \\
\hline -2 Log likelihood & $1.171,964$ & & $1.139,164$ & & $1.088,440$ & & $1.043,329$ & & $1.029,333$ & \\
\hline Observaciones & 2.104 & & 2.104 & & 2.104 & & 2.104 & & 2.104 & \\
\hline Chi-square & $56.420^{* *}$ & & $32.800^{* *}$ & & $50.724^{* *}$ & & $45.110^{* *}$ & & $13.996^{* *}$ & \\
\hline
\end{tabular}

$* \mathrm{p}<0,05 * * \mathrm{p}<0,01$ 
rés en las noticias $\left(\beta=0,481 ; 1,618 e^{x} ; p<0,05\right)$, la confianza en las noticias $\left(\beta=-0,278 ; 0,757 e^{x} ; p<0,05\right)$ y la percepción de independencia de los medios de comunicación ( $\beta=$ 0,$\left.450 ; 1,568 e^{x} ; p<0,05\right)$, la compra de un diario impreso es un predictor significativo y negativo $\left(\beta=-1,248 ; 0,287 e^{x} ; p<\right.$ $0,05)$. De esta forma, las personas que hayan comprado un periódico impreso tienen menos probabilidades de comprar un diario digital que las personas que no lo hayan comprado.

Finalmente, la sexta pregunta de investigación cuestionaba cómo afecta el uso de Facebook/Twitter al pago por un periódico digital. Además de la edad $\left(\beta=-0,027 ; 0,973 \mathrm{e}^{\mathrm{x}} ; \mathrm{p}<\right.$ $0,05)$, la educación $\left(\beta=0,217 ; 1,242 \mathrm{e}^{\mathrm{x}} ; \mathrm{p}<0,05\right)$, el interés en las noticias $\left(\beta=0,410 ; 1,508 e^{x} ; p<0,05\right)$, la confianza en las noticias $\left(\beta=-0,296 ; 0,744 e^{x} ; p<0,05\right)$, la percepción de independencia de los medios de comunicación $(\beta=0,469$; $\left.1,598 \mathrm{e}^{\mathrm{x}} ; \mathrm{p}<0,05\right)$, y la compra de un diario impreso $(\beta=$ -1,279; 0,278 $e^{x} ; p<0,05$ ), el uso de Twitter (pero no de Facebook) como herramienta informativa es un predictor significativo y negativo del pago por un diario digital $(\beta=$ $\left.-0,653 ; 0,520 e^{x} ; p<0,05\right)$. De esta forma, las personas que han usado Twitter para buscar, leer, ver, compartir o discutir contenido informativo tienen menos probabilidades de pagar por un periódico online.

La probabilidad de pago aumenta conforme mayor es la independencia percibida de los medios de comunicación

\section{Discusión}

El estudio confirma que el pago por noticias digitales es minoritario entre los internautas españoles $(10,2 \%)$ y que abunda el consumo gratuito de información. Mucho más relevantes y originales son los datos relacionados con los potenciales predictores del pago por noticias digitales. El análisis de los factores permite clasificarlos en tres grupos:

i) características sociodemográficas de la audiencia (sexo, edad, ingresos, educación y orientación política);

ii) factores actitudinales relacionados con la información (interés en la actualidad, confianza e independencia percibida);

iii) factores conductuales de consumo de información (compra de diario impreso y uso de redes sociales -Twitter y Facebook-como herramientas informativas).

Por lo que se refiere a los primeros, la edad (negativa), los ingresos y el nivel educativo tienen una asociación significativa con el pago por noticias, mientras que el sexo o la orientación política no, de tal forma que el público con menos edad, más ingresos y mayor nivel educativo tiene una mayor probabilidad de pagar por información digital. De esta forma, el segmento de mercado más joven, caracterizado por informarse casi exclusivamente a través de soportes digitales, tiene mayor probabilidad de pagar por noticias digitales que los más adultos, cuyo consumo de información online es complementario de los soportes impresos (Vara-Miguel, 2015). Por lo que se refiere al registro educativo, aquellos que tienen mayor nivel muestran una mayor probabilidad de pagar por noticias digitales. Este dato difiere de los obtenidos por algunos estudios previos que no encontraron relación alguna entre la educación y el pago por noticias digitales (Chyi; Lee, 2013; Punj, 2015) pero confirma las conclusiones de otras investigaciones (Chyi; Chadha, 2012), lo que sugiere la necesidad de seguir profundizando en la relación entre ambas variables, teniendo en cuenta que aquellas personas con mayor nivel educativo tienden a mostrar un mayor interés por la actualidad (Newman et al., 2016) y tienen una mayor necesidad de conseguir noticias de calidad por motivos profesionales (Punj, 2015).

En segundo lugar, todos los factores relacionados con la actitud del público hacia las noticias (el interés por la actualidad o el grado de confianza e independencia percibida en los medios) son fuertes predictores del pago. En este sentido, el estudio confirma, junto a estudios previos, la relevancia que tiene el interés en las noticias como predictor (Chyi; Chadha, 2012; Chyi; Lee, 2013; Goyanes, 2014). De idéntica forma, la probabilidad de pago aumenta conforme mayor es la independencia percibida de los medios de comunicación. Así, la gente tiene más inclinación al pago por aquello que considera bueno o valioso (Chyi; Lee, 2013). Si el pago se realiza siempre que el cliente considera que el beneficio esperado es superior al coste percibido de obtener un bien o servicio (Hsiao, 2011), y dado que la independencia informativa es un valor esperable de cualquier sistema de medios, es razonable pensar que los que perciben a los medios como independientes estén más dispuestos a pagar que los que creen que las empresas de comunicación son dependientes de grupos de interés políticos o económicos.

Dado que los lectores sólo pagan por aquello que consideran deseable, el pago por noticias de las que la gente no se fía cuestiona la intuición

Sin embargo, este razonamiento no se cumple con la confianza en los medios. Los datos muestran que aquellos que desconfían de las noticias tienen más probabilidad de pagar por información digital. Dado que los lectores sólo pagan por aquello que consideran deseable, el pago por noticias de las que la gente no se fía cuestiona la intuición. Sin embargo, cabe pensar que los que desconfían sí están dispuestos a pagar por aquellos medios concretos que merecen su confianza. Y que cuanto mayor es la desconfianza en general -porque mayor es la oferta gratuita de información-, más incentivos tienen para pagar por las noticias que les proporcionan aquellos medios dignos de su confianza. De alguna manera, la asociación entre desconfianza y pago estaría relacionada con el llamado "efecto tercera persona". Este efecto consiste en que las personas tienden a pensar que el resto de gente es más susceptible de ser influida negativamente por los medios que ellos mismos (Davison, 1983). Esta disparidad aplicada a la confianza en las noticias explicaría que hubiera personas con mayor tendencia a considerarse a sí mismas diferentes del resto o incluso inmunes (Perloff, 2009) y que creyeran que las únicas noticias fiables 
son aquellas que él/ella consume y por las que estaría dispuesto a pagar.

Un último aspecto a considerar tiene que ver con la influencia de los factores conductuales. Las dos variables analizadas -pago por un diario impreso y utilización de Twitter como medio informativo- apuntan la existencia de un efecto sustitución. Así, aquellos que pagan por un diario impreso tienen menos probabilidad de pagar digitalmente, de la misma forma que aquellos que usan Twitter para buscar, leer, ver o compartir noticias tienen menos probabilidad de pagar por un diario digital. Estos datos son coherentes con las características sociodemográficas ya analizadas: la venta de diarios impresos se da entre la población más adulta (y por lo tanto menos proclive al pago por noticias digitales, como se ha visto). Asimismo, los datos son coherentes con estudios previos que sugieren que la gente que usa Twitter habitualmente consulta más las noticias digitales que la media pero tiende a no pagar por la información (Goyanes, 2014). Las redes sociales son percibidas por sus usuarios como plataformas informativas gratuitas y su uso como canal informativo alternativo de los soportes tradicionales tiene un efecto negativo en la intención de pago por noticias digitales.

Las redes sociales son percibidas por sus usuarios como plataformas informativas gratuitas y su uso como canal informativo alternativo de los soportes tradicionales; tiene un efecto negativo en la intención de pago por noticial digitales

\section{Notas}

1. España es un mercado en el que la industria de los periódicos basa fundamentalmente sus ingresos por el pago del lector en la compra en quioscos (como es el caso de Reino Unido, Francia e Italia) en lugar de las suscripciones, como es el caso de Estados Unidos. Además, por ahora son muy limitadas las estrategias de pago por contenidos, sobre todo en relación con los países de nuestro entorno y a pesar de que España es uno de los países punteros en el uso de smartphones para uso y consumo informativo.

2. El trabajo de campo, comisionado por el Reuters Institute for the Study of Journalism - del que la Universidad de Navarra es socio académico- fue realizado por YouGov utilizando un cuestionario online a finales de enero y principios de febrero de 2016. Los datos fueron ponderados de acuerdo con los censos aceptados mayoritariamente por la industria para edad, sexo, región, lectura de prensa y nivel educativo con el fin de reflejar la población de España. La muestra es representativa de la población adulta española (mayores de 18 años) que tiene acceso a internet (77\% de la población). Como la encuesta trata sobre consumo de información, se excluyeron los casos de quien afirmó que no había consumido ningún tipo de noticias en el mes previo a la realización de la encuesta (un $3 \%$ de la muestra).
Las principales conclusiones del estudio del Reuters Institute for the Study of Journalism son de carácter descriptivo, es decir, únicamente se emplean estadísticos descriptivos para explorar la situación general del mercado español sin avanzar ningún análisis inferencial. El análisis efectuado y los resultados obtenidos y aquí expuestos son nuevos, salvo el dato descriptivo (bruto) en relación con la propia variable dependiente.

3. Los ingresos han dejado de ser estadísticamente significativos a partir de este modelo.

\section{Mención especial de reconocimiento}

Los resultados de este artículo forman parte del proyecto "Marcas tradicionales y nuevas marcas en los mercados de información online" (2014-16), financiado por el Plan de Investigación de la Universidad de Navarra (Piuna).

\section{Bibliografía}

Adams, Russell (2012). "Newspapers put faith in paywalls". The Wall Street Journal, 4 March 2012.

http://online.wsj.com/article/SB100014240529702038330 04577251822631536422.html

American Press Institute; ITZBelden (2009). Online revenue initiatives.

http://it.ejo.ch/wp-content/uploads/API-ITZBeldenRevenue-Initiatives-Survey1.pdf

Arrese, Ángel (2016). "From gratis to paywalls". Journalism studies, v. 17, n. 8, pp. 1051-1067.

https://doi.org/10.1080/1461670X.2015.1027788

Arrese, Ángel; Kaufmann, Jurg (2016). “Legacy and native news brands online: Do they show different news consumption patterns?". International journal on media management, v. 18, n. 2, pp. 75-97. https://doi.org/10.1080/14241277.2016.1200581

Berger, Benedikt; Matt, Christian; Steininger, Dennis M.; Hess, Thomas (2015). "It is not just about competition with 'free': Differences between content formats in consumer preferences and willingness to pay". Journal of management information systems, v. 32, n. 3, pp. 105-128. https://doi.org/10.1080/07421222.2015.1095038

Campos-Freire, Francisco (2010). "Los nuevos modelos de gestión de las empresas mediáticas". Estudios sobre el mensaje periodístico, v. 16, pp. 13-30.

http://revistas.ucm.es/index.php/ESMP/article/view/ ESMP1010110013A

Cea-Esteruelas, María-Nereida (2013). “Economía de los cibermedios: modelo de ingresos y fuentes de financiación". El profesional de la información, v. 22, n. 4, pp. 353-361. https://doi.org/10.3145/epi.2013.jul.12

Chiou, Lesley; Tucker, Catherine (2013). "Paywalls and the demand for news". Information economics and policy, v. 25, n. 2, pp. 61-69.

https://doi.org/10.1016/j.infoecopol.2013.03.001

Chyi, Hsiang-Iris; Chadha, Monica (2012). "News on new devices". Journalism practice, v. 6, n. 4, pp. 431-449. 
https://doi.org/10.1080/17512786.2011.629125

Chyi, Hsiang-Iris; Lee, Angela M. (2013). “Online news consumption". Digital journalism, v. 1, n. 2, pp. 194-211. https://doi.org/10.1080/21670811.2012.753299

Cook, Jonathan E.; Attari, Shahzeen Z. (2012). "Paying for what was free: Lessons from the New York Times paywall". Cyberpsychology, behavior, and social networking, v. 15, n. 12, pp. 682-687.

https://doi.org/10.1089/cyber.2012.0251

Davison, W. Phillips (1983). "The third-person effect in communication". Public opinion quarterly, v. 47, n. 1, pp. 1-15. https://doi.org/10.1086/268763

Filloux, Frederic (2011). "Analysing the metered model". Monday note, June 5.

https://mondaynote.com/analyzing-the-metered-modele6aa51ecdfc3

Goyanes, Manuel (2013). "Estrategias y modelos de negocio: Aclaración de conceptos y terminología de la prensa en internet". Estudios sobre el mensaje periodístico, v. 19, n. 1, pp. 419-431.

http://revistas.ucm.es/index.php/ESMP/article/view/42530

Goyanes, Manuel (2014). "An empirical study of factors that influence the willingness to pay for online news". Journalism practice, v. 8, n. 6, pp. 742-757.

https://doi.org/10.1080/17512786.2014.882056

Goyanes, Manuel (2015). "The value of proximity: Examining the willingness to pay for online local news". International journal of communication, v. 9, p. 18.

http://ijoc.org/index.php/ijoc/article/view/3388

Goyanes, Manuel; Dürrenberg, Catherina (2014). “A taxonomy of newspapers based on multi-platform and paid content strategies: evidences from Spain". International journal on media management, v. 16, n. 1, pp. 27-45.

https://doi.org/10.1080/14241277.2014.900498

Herbert, Jack; Thurman, Neil (2007). "Paid content strategies for news websites: An empirical study of British newspapers' online business models". Journalism practice, v. 1, n. 2, pp. 208-226.

https://doi.org/10.1080/17512780701275523

Himma-Kadakas, Marju; Kõuts, Ragne (2015). "Who is willing to pay for online journalistic content?". Media and communication, v. 3, n. 4, pp. 106-115.

https://doi.org/10.17645/mac.v3i4.345

Holm, Anna B. (2016). "Could freemium models work for legacy newspapers?". Nordicom information, v. 38, n. 1, pp. 83-87.

http://www.nordicom.gu.se/sites/default/files/kapitel-pdf/ nordicom-information_38_2016_1_83-87.pdf

Hsiao, Kuo-Lun (2011). "Why internet users are willing to pay for social networking services". Online information review, v. 35, n. 5, pp. 770-788.

https://doi.org/10.1108/14684521111176499

Huang, Edgard (2009). "The causes of youth's low news consumption and strategies for making youths happy news con- sumers". Convergence: The international journal of research into new media technologies, v. 15, n. 1, pp. 105-122. https://doi.org/10.1177/135 4856508097021

Jenner, Mike; Fleming, Ken (2011). "The push to paid: Attitudes of publishers toward paid content". Reynolds Journalism Institute, University of Missouri.

http://www.niemanlab.org/pdfs/rjipaidcontent.pdf

KPMG (2010). Anytime, anywhere. The rising demand on de the move.

https://es.scribd.com/document/45258922/AnytimeAnywhere-the-Rising-Demand-of-Media-on-the-Move

Newman, Nic; Fletcher, Richard; Levy, David A. L.; Nielsen, Rasmus K. (2016). Reuters Institute digital news report 2016. Reuters Institute for the Study of Journalism. University of Oxford.

http://reutersinstitute.politics.ox.ac.uk/publication/digitalnews-report-2016

Newman, Nic; Levy, David A. L. (2014). Reuters Institute digital news report 2014: Tracking the future of news. Reuters Institute for the Study of Journalism. University of Oxford.

http://reutersinstitute.politics.ox.ac.uk/publication/digitalnews-report-2014

Oh, Hyelim; Animesh, Animesh; Pinsonneault, Alain (2016). "Free versus for-a-fee: The impact of a paywall on the pattern and effectiveness of word-of-mouth via social media". MIS quarterly, v. 40, n. 1, pp. 31-56.

http://aisel.aisnet.org/cgi/viewcontent.cgi?article=3272\&context=misq

Peinado-Miguel, Fernando; Mateos-Abarca, Juan-Pablo (2016). Periodismo en el smartphone: monetización de aplicaciones para medios de comunicación. Icono14. Revista científica de comunicación y tecnologías emergentes, v. 14, n. 2, pp. 329-352.

https://doi.org/10.7195/ri14.v14i2.974

Pew Research Center (2011). "How people use tablets and what it means for the future of news". Pew Research Center. Journalism \& media, Oct. 25.

http://www.journalism.org/2011/10/25/tablet

Pew Research Center (2012). "How newspapers are faring trying to build digital revenue". Pew Research Center. Journalism \& media. March 5.

http://www.journalism.org/2012/03/05/search-newbusiness-model

Picard, Robert G. (2003). "Cash cows or entrecote: Publishing companies and disruptive technologies". Trends in communication, v. 11, n. 2, pp. 127-136.

https://doi.org/10.1207/S15427439TC1102_04

PricewaterhouseCoopers (2015). "Entertainment and media outlook 2015-2019. España".

http://informes.pwc.es/gemo

Punj, Girish (2015). "The relationship between consumer characteristics and willingness to pay for general online content: Implications for content providers considering subscription-based business models". Marketing letters, v. 26, n. 2, pp. $175-186$. 
https://doi.org/10.1007/s11002-013-9273-y

Rosenstiel, Tom; Mitchell Amy (2011). "Survey: Mobile news and paying online". The state of the news media 2011. Pew Research Center.

http://www.stateofthemedia.org/2011/mobile-survey

Sánchez-Tabernero, Alfonso (2008). "La prensa en Europa: claves de un sector estancado". Telos: Cuadernos de comunicación e innovación, v. 75, pp. 104-106.

https://telos.fundaciontelefonica.com/telos/articulocuaderno. asp@idarticulo=4\&rev=75.htm

Schlesinger, Philip; Doyle, Gillian (2015). "From organizational crisis to multi-platform salvation? Creative destruction and the recomposition of news media". Journalism: Theory, practice and criticism, v. 16, n. 3, pp. 305-323.

https://doi.org/10.1177/1464884914530223

Vara-Miguel, Alfonso (2015). “Complementariedad y canibalización en el mercado de diarios". En: Medina, Mercedes (coord.). La audiencia en la era digital. Madrid: Fragua, pp.

\section{7-216. ISBN: 9788470746703}

Vara-Miguel, Alfonso (2016): "Un 10\% de los internautas españoles pagó en 2015 por noticias digitales". Digital news report 2016: Cambios decisivos en el consumo de noticias digitales. http://www.digitalnewsreport.es/2016/un-10-de-losinternautas-espanoles-pago-en-2015-por-noticias-digitales

Vara-Miguel, Alfonso; Sanjurjo, Elena; Díaz-Espina, Carolina (2014). "Paid news vs free news: Evolution of the wsj.com business model from a content perspective (2010-2012)". Comunicacion and society, v. 27, n. 2, pp. 147-167.

http://www.unav.es/fcom/communication-society/es/ articulo.php?art_id $=494$

Zhang, Yue-Jeff; Nguyen, Dat-Dao (2004). "What makes consumers willing to pay for online services? Discovering consumer opinions and determinant factors on charged online services". Journal of internet commerce, v. 2, n. 4, pp. 35-53.

https://doi.org/10.1300/J179v02n04_03

\section{Inforảrea}

Ayudamos a tu organización en la transformación digital y el gobierno de la información

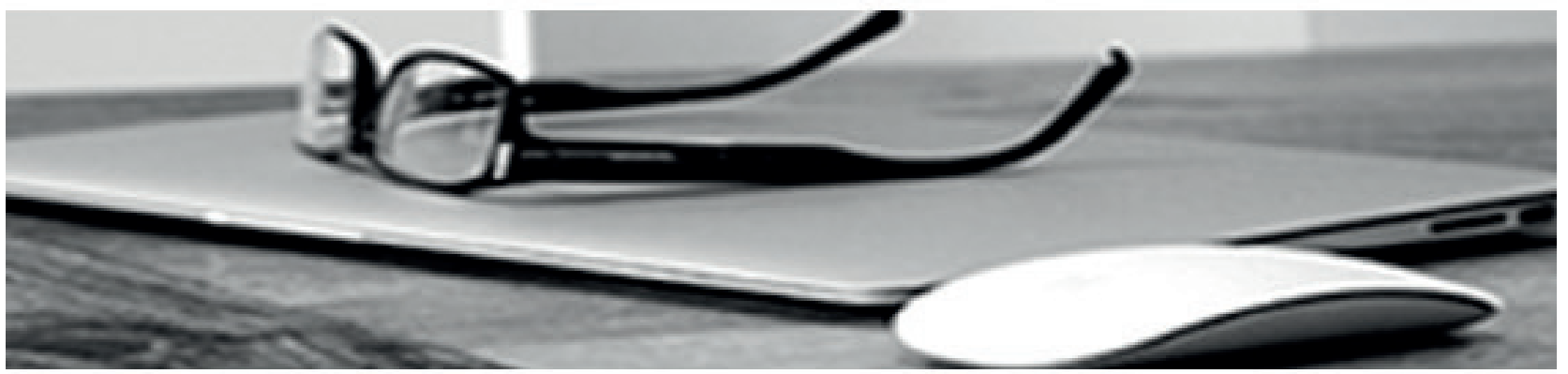

* Consultoría estratégica en gestión y gobierno de la información

* Gestión documental y "records management"

* Gestión de contenidos, intranets corporativas y entornos de colaboración

* Estudios especializados

Clientes satisfechos, cientos de empresas nacionales e internacionales y más de 30 años de experiencia son la mejor garantía de nuestra reputación. 